\title{
Design optimization method of line-of-sight with JACK for bulldozer operator
}

\author{
GUO Mei-ni ${ }^{1}$ \\ Department of the Ideological and Political Theory, Xi'an Physical Education University, Xi' an Shaanxi, 710068, China
}

\begin{abstract}
The objective of this article is to investigate the line-of-sight (LOS) and visual areas in order to provide a lower risk of accident and a best available view for the operator of industry machine. This paper presented a method of simulating an bulldozer operation in a virtual environment for the sake of analyzing LOS of the operator during conditions of load and unload respectively. Coverage zone tool in the JACK software was utilized to calculate the percentage of visible zones from the virtual operator's position. At the same time, the modification of design with additional cameras was proposed based on the results of LOS analysis, which significantly improved compared with original design. The results of this study proved that the modifications (with visual monitoring system) could improve visibility of bulldozer operators to some extent according to the optimization method.
\end{abstract}

\section{Introduction}

Work conditions of blue-collar are typically adverse, strenuous, and precarious conditions (e.g., monotonous, highly repetitive, long-duration sitting) [1]. At present, several studies have examined how to make operators more comfortable that mainly focused on the aspect of structural design [2] and accessories [3]. Nowadays, line-of-sight (LOS) issues for industry equipment is a growing focus of research. Patrick et al. [4] presented a method for assessment of degradation of task visibility from operator cabins of field machines. Alison Godwin et al. [5] studied on the implications of articulating machinery on operator line of sight and efficacy of additional camera locations. Three assessment test were utilized to identify forklift truck design factors for influencing visibility of operators [6]. As mentioned above, several studies have been done to improve the structure and shape of bulldozer. Hence, this paper investigated the potential utility of using computer simulation to evaluate image of line of sight and percentage of visible area during the operation of industry equipment. And some modifications to the visual area have been done to improve the LOS of operator in order to ensure workers' safety.

\section{Methods}

\subsection{Design procedures}

JACK software was offered by Siemens to analyze workplace environments with virtual people that can scale to match different population characteristics. It could be used to test designs and operations for a wide variety of human factors, including injury risk, timing, user comfort, reachability, lines-of-sight, energy expenditure, fatigue limits and other important parameters [11]. The coverage zone feature in JACK associated with the computer simulation program were utilized in this study enabled us to use obscuration zones.

Virtual computer analysis was integrated into the design process of industrial machines, and a design optimization method of LOS based on JACK was proposed to evaluate the potential use of a camera intervention on bulldozer. A flow chart describing the optimization procedure for LOS analyzing of operating movement was proposed (see Figure 1).

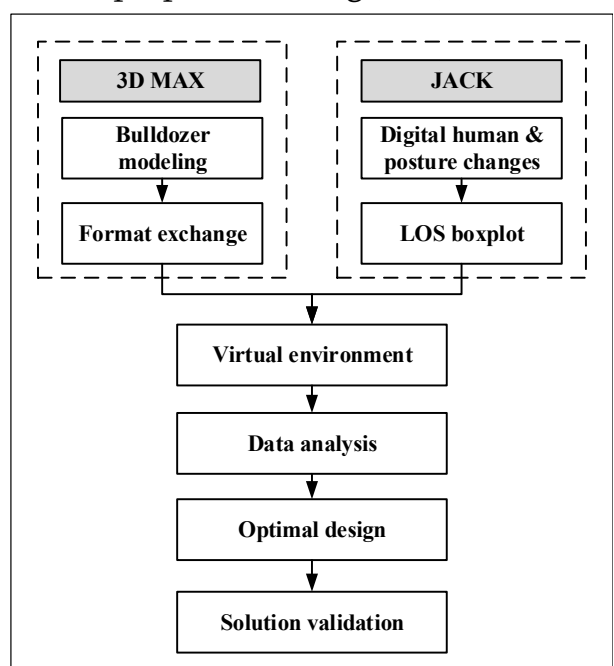

Figure 1. Flow chart of the study.

\footnotetext{
* Corresponding author: ${ }^{1957386089 @ \text { qq.com }}$
} 


\subsection{Vehicle design}

Developing animations of machines using the key frame method in Jack is time consuming [10]. JACK software is a ergonomic tool to assess and compute lines-of-sight impairments of operator, while there is a disadvantage to build 3D modelling. Thus, the accurate 3D files of bulldozer machine, which modelling by 3D MAX software, were imported into a computer simulation environment (JACK) as vrml format.

From the observation of the tasks carried out by bulldozer operators, there were a battery of operations including, in turn, digging, loading, driving, dumping. Observing working condition should be performed effectively in a short time. Two conditions, that is, load and unload should be taken into account. Therefore, the digital model of bulldozer is presented in Figure 2, illustrating the sequence of events and various operations in which investigation were made.

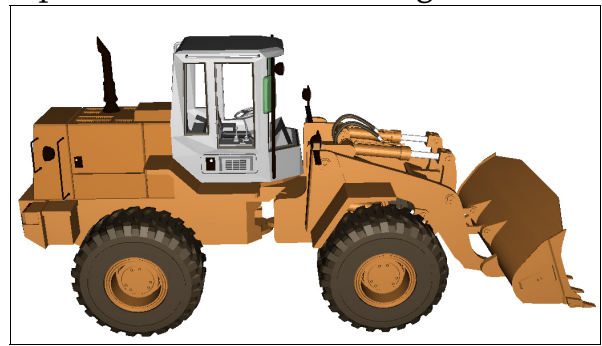

(a) Unload

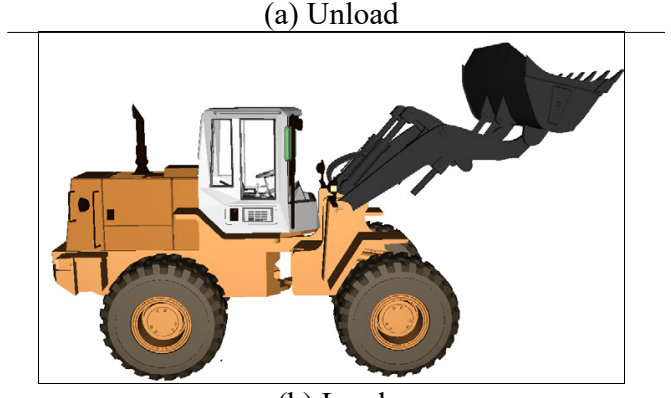

(b) Load

Figure 2. Digital model of bulldozer.

\subsection{Human models}

JACK software including nine digital human models[12]. Every default human figure has five percentile population, along with twenty-six body parameters to be chosen and adjusted depending on subjects' anthropometry. A 95th percentile male virtual human was chosen from CHINESE database, as a bulldozer operator, to simulate interactive operation tasks in which 3D models of bulldozer.

\subsection{LOS boxplot}

The LOS boxplot provides visual and non-visible images on coverage plane respectively, in which red indicating non-visible zones and green indicating visible zones. The program calculated the percentage of area that is visible/non-visible to the operator. LOS boxplot has been used previously in studies evaluating of LOS impairments in industrial machines [13].

The present study used the method already conceived by Jeffkins et al. [9]. Coverage planes representing visual attention locators for any vehicle under any operating scenario could be evaluated with this method. A rectangular box areas with $20 \mathrm{~m}$ width, $10 \mathrm{~m}$ length and $4.5 \mathrm{~m}$ height was generated by utilizing coverage tool, and it was divided into 11 separate panels in which a $0.1 \mathrm{~m}$ nodal space was set up.

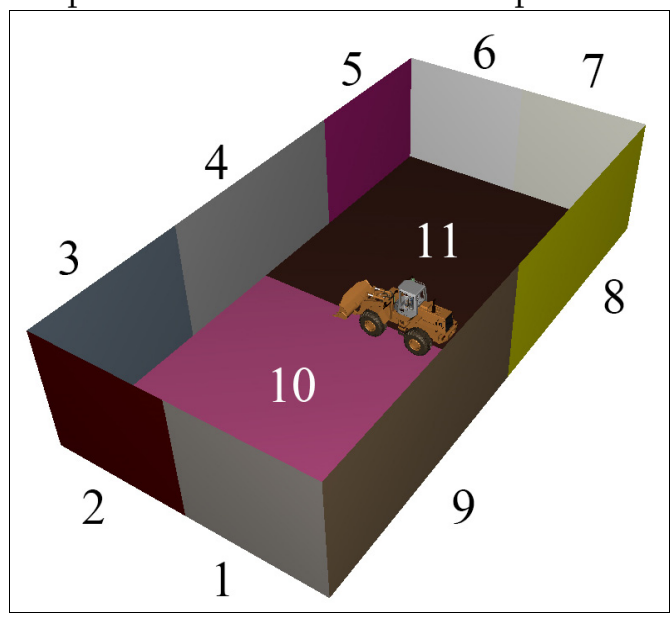

Figure 3. A LOS boxplot divided into 11 separate panels.

\section{Results and design optimization}

\subsection{Data analysis}

The data collection for the computer simulation method was performed by using the coverage zone tool in the JACK program to determine LOS from the virtual operator's position. Figure 4 displayed the image about which LOS of operator are to be made in condition load and unload operating, as it showed the green and red areas were behalf of visible and nonvisible zones respectively.

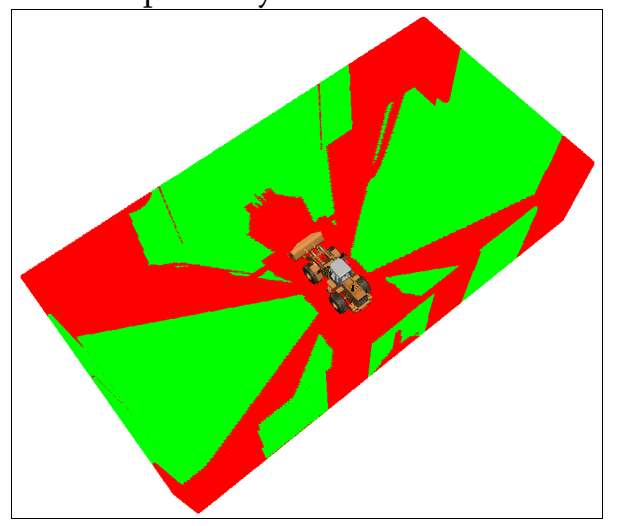

(a) Unload 


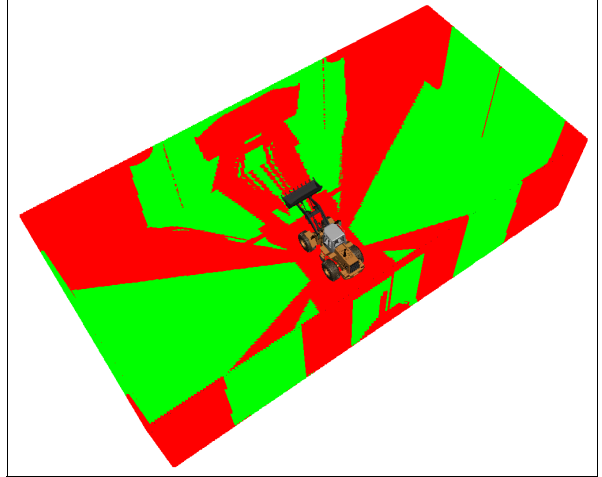

(b) Load

Figure 4. Restricted LOS for original design.

\subsection{Design optimization}

As shown above, the front and back view of operator was primarily obscured by rear-view mirror and structures of cabin under unload condition, and bucket principally restricted the front of LOS severely under load condition. Therefore, after above testing, the bulldozer design was modified based on the results of line-of-sight analysis. Compared structure of original and modified bulldozer, given as below, were illustrated in Figure 5. First of all, the dimensions of rear-view mirrors were narrowed. Secondly, the parts of support structure of original bulldozer were redesigned as well. Thirdly, the placement of four searchlights were relocated. Finally, the frame of windows were adjusted slightly. All of which were non-changing with original structure of the bulldozer cab.

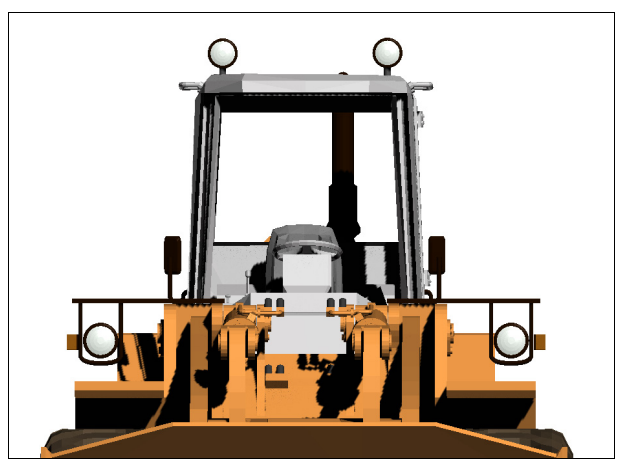

Figure 5. Figures of modified bulldozer.

The LOS boxplot and data presented in Figure 4 demonstrated that the operator had virtually no visual information available from the front of view when loading due to bucket and rack mainly. On the other hand, the severely restricted areas of back-side resulting from power system and balance weight that were loaded on the rear of vehicle. Hence, other than improving the original bulldozer as mentioned above (fig. 5), visual monitoring system was also installed on the front and rear side of modified vehicle in order to ameliorate the line-of-sight as fig. 6 depicted. The monitoring system was able to provide more visual information to operator towards front and rear side of vehicle. Consequently, reductions in pilot errors and improvements of decision-making with low accident rate will be made by operator [14].

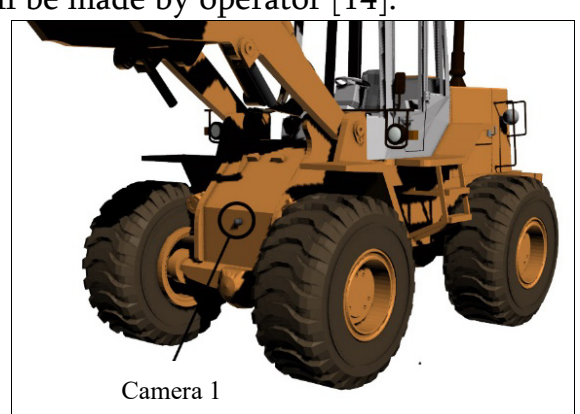

(a) Camera 1

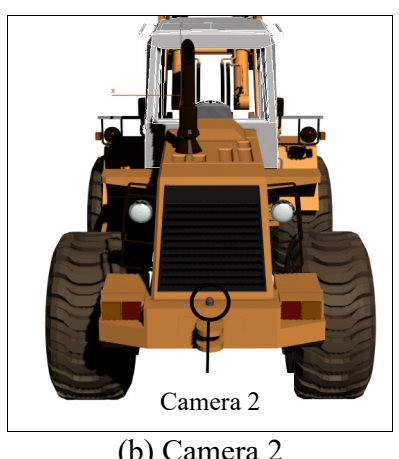

Figure 6. Figures of original and modified bulldozer.

The 3-D file of modified industry machine was imported into JACK software and the LOS boxplot analysis of unload and load condition were repeated one more time to give best assessment.

Data collection was performed for original and modified design with two camera perspectives by using the coverage zone tool in JACK to analyze LOS area of operator (Table 1).

Comparison of LOS boxplot area for the original and modified design were presented in Table 2 and Figure 7. In the present study it was proved that, in general, the modified design of bulldozer with camera system provided more than $80 \%$ visual area of LOS in these key zones under both operating conditions (unload and load) with the exception of ground-level LOS (zones 10 and 11).

Table 1. Percentage of LOS boxplot area visible for original and modified design with cameras.

\begin{tabular}{|c|c|c|}
\hline & Zones (\%) & Area \\
$\left(\mathrm{m}^{2}\right)$
\end{tabular}




\begin{tabular}{|c|c|c|c|c|c|c|c|c|c|c|c|c|}
\hline & 1 & 2 & 3 & 4 & 5 & 6 & 7 & 8 & 9 & 10 & 11 & \\
\hline Unload- modified & 75.43 & 97.91 & 30.71 & 98.28 & 33.96 & 99.31 & 85.15 & 47.03 & 53.38 & 59.98 & 59.11 & 297.62 \\
\hline Load- modified & 74.87 & 98.01 & 29.13 & 53.48 & 36.98 & 99.23 & 77.30 & 47.60 & 52.73 & 58.62 & 59.98 & 279.47 \\
\hline Camera 1 & 1.14 & 22.87 & 87.34 & 98.29 & 88.32 & 22.54 & 1.15 & 0.00 & 0.00 & 17.01 & 19.03 & 129.59 \\
\hline Camera 2 & 96.14 & 3.33 & 0.00 & 0.00 & 0.00 & 0.88 & 92.38 & 98.38 & 98.95 & 8.11 & 8.06 & 148.33 \\
\hline
\end{tabular}

Table 2. A comparison for percentage of LOS boxplot area visible with original and modified design.

\begin{tabular}{|c|c|c|c|c|c|c|c|c|c|c|c|c|c|}
\hline & \multicolumn{11}{|c|}{ Zones (\%) } & \multirow{2}{*}{$\begin{array}{l}\text { Area } \\
\left(\mathrm{m}^{2}\right)\end{array}$} & \multirow{2}{*}{$\begin{array}{c}\text { Ratio } \\
(\%)\end{array}$} \\
\hline & 1 & 2 & 3 & 4 & 5 & 6 & 7 & 8 & 9 & 10 & 11 & & \\
\hline $\begin{array}{l}\text { Unload- } \\
\text { modified }\end{array}$ & 96.14 & 97.91 & 30.71 & 98.28 & 33.96 & 99.31 & 92.38 & 98.38 & 98.95 & 59.98 & 59.11 & 347.52 & 73.94 \\
\hline $\begin{array}{c}\text { Load- } \\
\text { modified }\end{array}$ & 96.14 & 98.01 & 87.34 & 98.29 & 88.32 & 99.23 & 92.38 & 98.38 & 98.95 & 58.62 & 59.98 & 377.01 & 80.21 \\
\hline $\begin{array}{l}\text { Load- } \\
\text { original }\end{array}$ & 72.86 & 60.97 & 24.75 & 50.25 & 19.05 & 54.50 & 75.26 & 39.46 & 43.50 & 52.95 & 54.22 & 233.73 & 49.73 \\
\hline
\end{tabular}

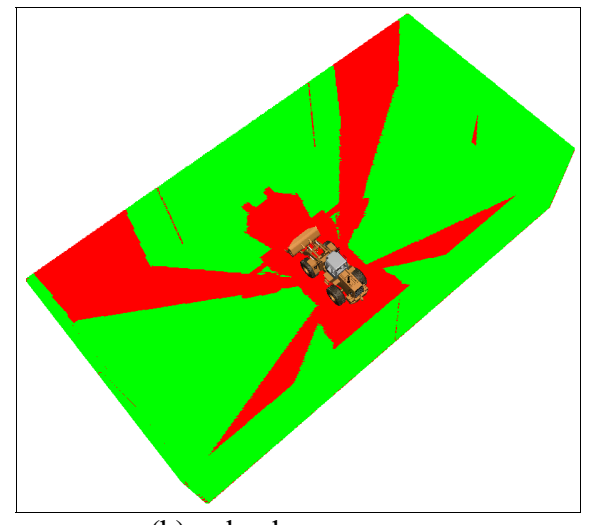

(b) unload

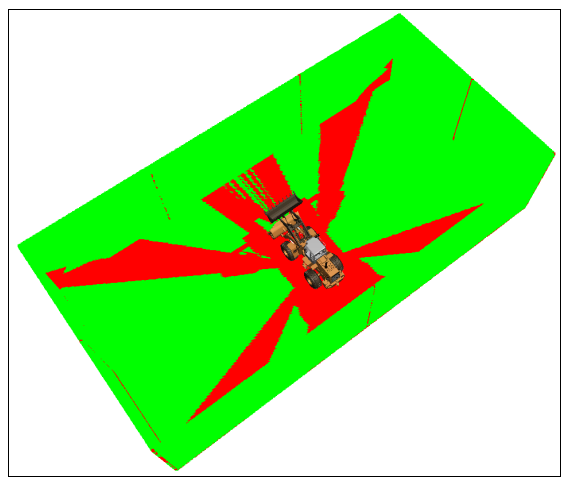

(b) load

Figure 7. LOS boxplot area for modified design.

\section{Conclusions}

This paper has presented a method of developing an integrated system including modeling software (3D MAX software) and LOS boxplot assessment (JACK software) that enabled researchers to objectively investigate the visual monitoring system locations on an industry machine that could best improve LOS of operators. As described, the coverage plane tool could preferably provided both a visual and a quantitative method of LOS evaluation. With reference to the results of the percentage of visible area at various zones, a targeted optimization was performed for improving the visible areas of a bulldozer. The methods discussed in this paper presented the recommendations for the improvement of ergonomic aspects of product design of the industry machines, so as to offering the best LOS for potential vehicle operator.

\section{Acknowledgment}

Fund Project: Shaanxi Culture Creative Industry Innovation policy Research (2018 Shaanxi Science and Technology Plan Soft Science Research Project) 


\section{References}

1. O. HÃOmmig. Front. Public Heal. 2, (2014)

2. X. Yu, L. Lijuan. Constr. Mach. Equip. 47, 23-26,7 (2016)

3. J. Youchang. Des. \& Manu. 4, 248-52 (2015).

4. P.J. Barron, P.M.O. Owende, K.P. McDonnell, S.M. Ward. Int. J. Ind. Ergon. 35, 665-73 (2005)

5. N. Schwabe, A. Godwin. Safety 3, 17 (2017)

6. C.B.Choi, P. Park, Y.H. Kim, M. Susan Hallbec, M.C. Jung. Appl. Ergon. 40, 280-5 (2009)

7. L. Sheming, W. Xiaoping, C. Dengkai, W. Shuxia. Comput. Mod. 8, 106-10 (2013)

8. T. Zhengwen, X. Hongjun, S. Rune. Aeronaut. Comput. 40, 79-81,106 (2010)

9. A. Jeffkins, T. Eger, A. Salmoni, R. Whissell. Ergon. Des. 12, 12-7 (2004)

10. S.C. Puthenveetil, C.P. Daphalapurkar, et al. Virtual Real. 19, 119-28 (2015)

11. Siemens PLM Software. https://www.plm.automation.siemens.com/en/products (2018)

12. Y. Qingsong, L. Qianfang, Z. Qianxiang, et al. Sp. Med. \& Med. Eng. 29, 440-5 (2016)

13. A. Godwin, T. Eger. Int. J. Ind. Ergon. 39, 146-51 (2009)

14. Y. Ke, G. Mingyu, C. Jifeng. J. Hangzhou Dianzi Univ. 27, 1-4 (2007) 